\title{
A CENARIZAÇÃO E O MUNICÍPIO DE PRESIDENTE PRUDENTE/SP: MÉTODOS PROPECTIVOS E OS ATORES SOCIAIS
}

Ivan Márcio Gitahy Junior

Universidade do Oeste Paulista - UNOESTE, curso de Administração, Presidente Prudente, SP. E-mail: ivan@unoeste.br

\section{RESUMO}

Com o intuito de discutir oportunidades e ameaças, pontos fortes e fracos relacionados a uma determinada região considerando seu planejamento, gestão e desenvolvimento faz-se necessário estudos sobre prospecção considerando cenários. A partir desta afirmativa, a pesquisa objetiva gerar e analisar cenários prospectivos para o Município de Presidente Prudente/SP até 2030 a partir da visão de atores sociais. Para a realização da pesquisa tomar-se-á como metodologia técnicas de construção de cenários prospectivos como: Godet, Global Business Network, Porter, Delphi, Matriz de Impactos Cruzados, Teorema de Bayes, Simulação Monte Carlo, ferramentas que compõem o método Grumbach. Com essa metodologia será possível, além de se elaborar cenários, identificar quais deles serão mais motrizes e dependentes e como se comportarão na matriz dos impactos cruzados. Cabe lembrar que tal matriz permite o cálculo dos graus de motricidade e dependência onde, quanto maior for o grau de motricidade de um evento, maior influência terá ou não nas probabilidades dos outros eventos. Com o exposto, tal pesquisa busca compreender e contextualizar o Município de Presidente Prudente/SP a luz de seu planejamento, considerando a participação de atores sociais locais.

Palavras-chave: Cenários. Planejamento. Presidente Prudente/SP

\section{SCENARIO BUILDING AND THE MUNICIPALITY OF PRESIDENTE PRUDENTE / SP: PROACTIVE METHODS AND SOCIAL ACTORS}

\begin{abstract}
In order to discuss opportunities and threats, strengths and weaknesses related to a particular region considering its planning, management and development, it is necessary to study prospecting considering scenarios. From this point of view, the research aims to generate and analyze prospective scenarios for the Municipality of Presidente Prudente / SP until 2030 from the perspective of social actors. In order to carry out the research, it will be used as a methodology for the construction of prospective scenarios such as: Godet, Global Business Network, Porter, Delphi, Cross-Impact Matrix, Bayes Theorem, Monte Carlo Simulation, Grumbach method tools. With this methodology it will be possible, besides elaborating scenarios, to identify which ones will be more motivated and dependent and how they will behave in the cross impact matrix. It should be remembered that such matrix allows the calculation of the degrees of motricity and dependence where, the greater the degree of motricity of an event, the greater influence it will have or not in the probabilities of other events. With the above, this research seeks to understand and contextualize the Municipality of Presidente Prudente / SP in the light of its planning, considering the participation of local social actors.
\end{abstract}

Keywords: Scenarios. Planning. Presidente Prudente / SP

\section{INTRODUÇÃO/ JUSTIFICATIVA}


Os estudos prospectivos representam uma importante ferramenta para discutir oportunidades e riscos quanto ao planejamento estratégico e gestão de um dado Município. A partir desta afirmativa, a presente pesquisa tem como objetivo gerar e analisar cenários prospectivos para o Município de Presidente Prudente/SP até 2030 a partir da visão de atores sociais na elaboração de eventos.

O cenário da pesquisa acontecerá na cidade de Presidente Prudente, denominada Capital da Alta Sorocabana. Segundo os últimos dados da Fundação Seade fundação, datado de 2017, o Município de Presidente Prudente/SP conta com uma área de 560,64 km2, a sua densidade demográfica (habitantes por km2) é de 387,57 e o grau de urbanização em \% é de $97,96 \%$.

Acredita-se que além da relevância social e empresarial que os resultados da presente pesquisa podem trazer a Presidente Prudente/SP, também haverá uma ampliação teórica e empírica dos estudos sobre o método de cenários prospectivos pode auxiliar na compreensão de um dado Município paulista, considerando seu planejamento e gestão. A fim de entender melhor o que se busca com o trabalho, será apresentado o referencial teórico sobre cenários prospectivos e alguns conceitos de desenvolvimento e crescimento. Com a pesquisa de campo, ter-se-á uma análise dos cenários prospectivos do Município de Presidente Prudente/SP até o ano de 2030.

Segundo os autores Khan e Wierner (1967), a preocupação com o futuro liga-se a cinco fatores de interesse, ou seja: o lucro; a taxa de mudança; a necessidade filosófica, ou seja, o desejo de saber para onde ir; as questões militares, para onde iria o mercado bélico e o modismo do futuro. Seja qual for o fator que impulsiona o conhecimento do futuro, o homem acredita que estudar cenários pode auxiliar a encontrar as respostas desejadas.

Os métodos de construção de cenários têm como objetivo apresentar alternativas que levem a prospecções, buscando destacar não só tendências e ocorrências dominantes, mas também a possibilidade de transformação do ambiente. Tais estudos, segundo Amara (1988) e Coates (1994) apresentam uma natureza exploratória e uma abordagem interdisciplinar na pesquisa sobre os rumos e as possibilidades do futuro.

$O$ estudo com cenários prospectivos abrange diversas áreas e a temática prospecção ganhou destaque no século XX e XXI, principalmente a partir do ano de 1970 com a crise da energia, onde o desejo de conhecer o futuro está presente desde os primórdios da humanidade, mas ainda sem a metodologia dos cenários prospectivos.

Do ponto de vista metodológico a pesquisa foi fundamentada, no primeiro momento, em produções científicas sobre o tema proposto. Iniciou-se, então, uma busca pela biblioteca digital de teses e dissertações (IBICT).

Para o estabelecimento do referencial teórico sobre os métodos de construção de cenários fundamentados foram consultados um amplo conjunto de autores que vêm se dedicando ao tema nas últimas décadas, dentre os quais, destacam-se: Berger (1964); Kahn (1969); Rattner (1979), Wack (1985); Wright e Guimarães (1986); Porter (1992; 1998); Amara (1988); Coates (1994); Godet (1993;2000); Glenn (1994); Johnson e Marcovitch (1994); Mason(1994); Schoemaker (1995); Schwartz (2000); Ringland (1998); Marcial (2002, 2008); Jaguaribe (2000); Araújo (2001); Schwartz (2000); Almeida (2002); Wright e Giovanazzo (2000; 2006); Porto (2001); Bethlem (2002); Buarque (2003); Heijden (2004); Wright (2005); Franco (2007); Grumbach (2002); Marcial e Grumbach (2008).

Aliando a ideia de prospecção a cenários, os autores Kahn e Wierner (1967) foram os primeiros a popularizar, a partir de sua obra The Year 2000, a ideia de cenários prospectivos.

O termo cenário caracteriza-se por um estudo criativo sobre uma situação futura utilizando uma metodologia própria, onde a construção de respostas está diretamente ligada às transformações que ocorrem no ambiente. 
Para alcançar os objetivos propostos será elaborada uma revisão bibliográfica sobre o tema crescimento e desenvolvimento tendo em vista a elaboração de um referencial teórico adequado para a pesquisa. Os principais autores consultados foram: Keynes (1990), Samuelson e Nordhaus (2004), Smith (1996), Ricardo (1996), Karl Marx (1987), Schumpeter (1988, 1982), Sandroni (1999), Furtado (1974), Romero (2000), Breda e Andrade (2002), Osberg e Sharp (1998) e Amaral Filho (1996).

Segundo Amaral (1996), desenvolvimento deve ser pensado a partir dos próprios atores sociais locais e é a eles que vamos dar voz a fim de se entender o cenário atual e de prospecção do objeto de pesquisa, ou seja a cidade de Presidente Prudente/SP.

Aliando as bases teóricas de cenários prospectivos e desenvolvimento a presente pesquisa além de se justificar socialmente, pois o planejamento estratégico do Município de Presidente Prudente/SP pode ter mais subsídios com os cenários prospectivos de desenvolvimento, justificase cientificamente tendo em vista que na área acadêmica não há estudos sobre a questão da prospecção do desenvolvimento do Município de Presidente Prudente/SP a partir dos atores sociais. Considera-se isto fundamental pois ao estudar o desenvolvimento, faz-se um estudo transdisciplinar tendo em vista que o mesmo deve envolver: ser humano, economia, meio ambiente e inovação. A pesquisa terá como a construção e análise de cenários prospectivos para o Município de Presidente Prudente/SP até 2030 a partir da visão dos atores sociais na elaboração de eventos.

\section{METODOLOGIA}

Para a realização desta pesquisa tomar-se-á como metodologia diversas técnicas de construção de cenários prospectivos como: Godet, Global Business Network, Porter, Delphi, Matriz dos Impactos Cruzados, Teorema de Bayes, Simulação Monte Carlo, ferramentas que compõem o método Grumbach. Com essa metodologia de pesquisa será possível, além de se elaborar cenários, identificar quais deles serão mais motrizes e dependentes e como se comportarão na matriz dos impactos cruzados. Cabe lembrar que tal matriz permite o cálculo dos graus de motricidade e dependência onde, quanto maior for o grau de motricidade de um evento, maior influência terá ou não nas probabilidades dos outros eventos. Quanto maior o grau de dependência maior a probabilidade da ocorrência sofrer influência dos demais eventos.

A presente pesquisa se desenvolveu numa abordagem quali-quantiativa, tomando como principal locus de investigação, o Município de Presidente Prudente/SP. Desta maneira será feito um levantamento procurando identificar, neste ambiente, alguns atores sociais que pudessem auxiliar a pesquisa. A escolha dos atores sociais entrevistados, moradores do Município de Presidente Prudente/SP, será feita considerando o critério aceite em participar da pesquisa e ser do segmento governo municipal; empresariado; universidade; lideranças sociais e trabalhadores. De cada segmento serão entrevistados dois membros, totalizando cinco segmentos e dez entrevistados. Os contatos dos atores sociais serão obtidos por meio de pesquisa na rede mundial de computadores. Dos resultados obtidos, far-se-á um primeiro contato com os possíveis entrevistados através de e-mail. O referido e-mail fará menção a pesquisa e apresentará, aos respondentes, a importância do estudo. Com o aceite, o entrevistado assinará o termo de consentimento livre e esclarecido ${ }^{1}$ e as entrevistas ${ }^{2}$ serão agendadas.

A construção dos cenários prospectivos será baseada no método de Grumbach amparada em várias técnicas e métodos como: Godet, Global Business Network, Porter, Delphi, Teorema de Bayes, Simulação Monte Carlo. 
Método Godet relacionado a técnica com a administração estratégica e baseia-se em seis etapas, a saber: Delimitação do sistema e do ambiente; Análise estrutural do sistema e do ambiente; Seleção dos condicionantes do futuro; Geração de cenários alternativos; Teste de consistência, ajuste e disseminação; Opções estratégicas e planos/monitoração estratégica.

O método Global Business Network (GBN) realiza planejamento estratégico para as empresas anglo-saxônicas e baseia-se em 8 etapas, a saber: Identificação da questão principal; Identificação das principais forças do ambiente local; Identificação das forças motrizes; Ranking por importância e incerteza; Seleção das lógicas dos cenários; Descrição dos cenários; Análise das implicações e opções; Seleção dos principais indicadores e sinalizadores.

Já o método Porter a construção de cenários é uma das mais importantes ferramentas para se desenvolver estratégias relacionadas às incertezas do futuro. Seguem as oito etapas: Propósito do estudo; Estudo histórico e da situação atual; Identificação das incertezas críticas; Comportamento futuro das variáveis; Análise de cenários e consistência; Concorrência; Elaboração e histórias de cenários; Elaboração das estratégias competitivas.

Entende-se por Método Delphi, uma metodologia onde especialistas são consultados, várias vezes, por meio de entrevistas individuais para que o grupo possa chegar a uma reflexão convergente sobre os possíveis cenários criados sobre as incertezas críticas.

O Teorema de Bayes demonstra a relação entre uma probabilidade condicional e a sua inversa; as suposições ou a opinião de várias pessoas sobre o relacionamento de fatos alegados e uma hipótese pode determinar como estas pessoas utilizam estes fatos como evidência.

E a Simulação de Monte Carlo é uma técnica matemática computadorizada, onde por conta de planilhas eletrônicas, considera o risco em análises quantitativas e tomadas de decisão podendo ser utilizada em diversas áreas. Sua utilização é uma alternativa bastante razoável, capaz de reduzir a incerteza, sem adição significativa de custo e tempo, necessitando, apenas, conhecimentos estatísticos para leitura dos resultados.

\section{DISCUSSÃO}

Após a realização da pesquisa com os atores locais pode-se, com esse método, chegar à definição de estratégias adequadas para o desenvolvimento do Município de Presidente Prudente/SP.

De acordo com Marcial e Grumbach (2002), com os cenários traçados e seus diversos acompanhamentos, tem-se uma visão sistêmica e alinhada, conseguindo compreender os acontecimentos. Segundo os autores citados, prever o futuro não é objetivo dos estudos prospectivos, mas sim estudar as diversas possibilidades de futuros plausíveis.

Comungando de tal filosofia, a construção de cenário auxiliará nos desdobramentos das incertezas quanto ao desenvolvimento e crescimento do Município de Presidente Prudente/SP até 2030, bem como auxiliará na compreensão e contextualização do Município de Presidente Prudente/SP a luz do planejamento, desenvolvimento e de seu crescimento considerando a participação de atores sociais locais.

A discussão teórica foi de fundamental importância para se refletir sobre a condição municipal considerando crescimento, desenvolvimento econômico e desenvolvimento humano a luz, não só de autores clássicos como também contemporâneos, apresentados na pesquisa.

\section{RESULTADOS}

Tal método gerará cenários, como: mais provável, ideal e tendência. O cenário mais provável deve ser entendido com o resultado proveniente da opinião dos peritos caracterizando a aplicação do método Delphi. O cenário ideal deve ser entendido como o mais adequado, ou seja, o melhor para o Município de Presidente Prudente/SP objeto da pesquisa, de acordo com a opinião 
dos peritos. O cenário de tendência representa um cenário prospectivo gerado, a partir do grupo de controle, considerando uma continuidade dos acontecimentos atuais sobre o futuro.

O cenário mais provável acontecerá se todos os eventos ocorrerem, por ser entendido como o resultado proveniente da opinião dos peritos caracterizando a aplicação do método utilizado. O cenário ideal deve ser entendido como o mais adequado e acontecerá se não houver Crescimento populacional do Município de Presidente Prudente, tendo sido identificado como medias e consequências, a favelização. O cenário tendência ocorrerá caso o evento que menciona a Redução da taxa de analfabetismo da população adulta ocorrer, pois foi o evento que os peritos consideraram como um forte índice de crescimento e desenvolvimento.

\section{CONCLUSÃO}

Com o referencial teórico apresentado sobre cenários prospectivos incluindo o método de construção, Grumbach, foi possível identificar a necessidade de se conhecer o futuro das cidades e de entender, a partir de especialistas, chamados aqui de peritos, como esse futuro poderia ser gerado. Com este estudo fez-se um ensaio sobre os cenários prospectivos não só para o município de Presidente Prudente/SP, mas para cidades, contribuindo para que as mesmas consigam ter uma visão de futuro considerando planejamento, gestão e desenvolvimento.

Os cenários devem representar um panorama do município de Presidente Prudente/SP considerando sua retrospecção, conjuntura atual e prospecção. Esse conjunto de variáveis corrobora com os objetivos da pesquisa que é gerar e interpretar cenários prospectivos a luz do crescimento, desenvolvimento, planejamento e gestão em um determinado horizonte temporal.

\section{REFERÊNCIAS BIBLIOGRÁFICAS}

AMARA, R. What we have learned about forecasting and planning. Futures, v. 20, n. 4, p.385-401, 1988.

AMARAL FILHO, J. Desenvolvimento regional endógeno em um ambiente federalista. In: Planejamento e políticas públicas. Brasília, IPEA, n. 14. dez. , 1996

BERGER, G. Phénoménologie du temps et prospective. Paris: PUF, 1964.

COATES, J. F.; MAHAFFIE, J. B.; HINES, A. Technological Forecasting: 1970-1993. TechnologicalForecastingand Social Change, v.47, p.23-33, 1994

GODET, M. A Caixa de Ferramentas da Prospectiva Estratégica - Problemas e métodos. Lisboa: Cadernos do Centro de Estudos de Prospectiva e Estratégia, 2000.

GRUMBACH, R. J. S. Cenários Prospectivos - A Chave para o Futuro: Planejamento Estratégico. Rio de Janeiro: Ed. Catau, 2008.

JOHNSON, B. B.; MARCOVITCH, J. Uses and Applications of Technology Futures in National Development: the Brazilian Experience. Technological Forecasting and Social Change, v.45, p.1-30, 1994.

JOHNSON, B.; WRIGHT, J.; GUIMARÃES, P. Prognósticos tecnológicos como atividade complementar do planejamento: a experiência do CENPES em águas profundas. São Paulo: Fundação Instituto de Administração (FIA), 1986 
KAHN, H. WIENER, A.J. The year 2000: a framework for speculation on the next thirty-three years. s/l: Hudson Institute, 1968

MARCIAL, E. C., GRUMBACH, R. J. S. Cenários Prospectivos - Como Construir um Futuro Melhor. Rio de Janeiro: Ed. FGV, 2008.

PORTER, M. E. . Vantagem Competitiva. Rio de Janeiro: Campus, 1989

SCHUMPETER, J. Teoria do desenvolvimento econômico. - São Paulo: Abril Cultural, 1982.

WRIGHT, J. T. C.; GIOVINAZZO, R. A. DELPHI - Uma ferramenta de apoio ao planejamento prospectivo. Caderno de Pesquisas em Administração, São Paulo, v. 1, n. 12, p. 54- 65, 2000. 